\title{
Teología de la Liberación: influencias, características y las tensiones con el Vaticano
}

Liberation theology: influences, characteristics and tensions with the Vatican

\author{
Por: González, Daiana Marlén ${ }^{*}$ \\ Universidad Nacional del Nordeste. \\ E-mail: marlengonzalez9679@gmail.com
}

Fecha de recepción: 08/04/2021

Fecha de aprobación: 25/04/2021

DOI: http://dx.doi.org/10.30972/ach.065601

\section{Resumen}

El surgimiento y el desarrollo de la Teología de la Liberación en América Latina supuso una importante novedad para la Iglesia Católica y para la Teología tradicional. A los teólogos de la liberación se les reconoce por su gran compromiso pastoral, social y político. La Teología de la Liberación se presenta como una alternativa de interpretación bíblica y de vida cristiana desde la experiencia de los países latinoamericanos. Se orienta también a promover un mayor compromiso y participación en los procesos políticos de liberación de estos países y, por esta razón, lleva a cabo la revisión crítica del rol que la Iglesia debe tomar al respecto. Es conocida por su opción en favor de los pobres y los oprimidos a quienes considera los principales perjudicados por la injusticia y pobreza que atraviesa Latinoamérica.

Lo anterior no siempre ha sido bien recibido por los clérigos más ortodoxos, quienes vieron en sus ideas un fuerte sesgo marxista. Por ello, en torno a la Teología de la Liberación se han generado grandes disputas, muchas de ellas aún vigentes. En este trabajo nos proponemos retomar una de esas disputas mediante el análisis de dos textos que son claves para comprender al movimiento teológico y las polémicas que se suscitaron en el interior de la Iglesia Católica. El escrito se desarrollará a partir de una

\footnotetext{
*Estudiantx de la Licenciatura en Filosofía de la Facultad de Humanidades (UNNE).
} 
lectura del Documento Conclusivo de la Segunda Conferencia del Episcopado Latinoamericano (1968) y de la Instrucción sobre algunos aspectos de la "Teología de la Liberación" (1984) del Cardenal Joseph Ratzinger, quien intentó advertir sobre las posibles "desviaciones" presentes en la Teología de la Liberación.

Palabras claves: Teología- Liberación-Vaticano-Marxismo-Latinoamérica.

\section{Abstract}

The emergence and development of Liberation Theology in Latin America was a novelty for the Catholic Church and for traditional theology. Liberation theologians are recognized for their great pastoral, social and political commitment. Liberation Theology is presented as an alternative of biblical interpretation and Christian life from the experience of Latin American countries. It also aims at promoting greater commitment and participation in the political processes of liberation in these countries, and for this reason, carries out a critical review of the role that the Church must take in this regard. It is known for its choice for the poor and oppressed whom it considers the main victims of the injustice and poverty that Latin America is experiencing.

This has not always been well received by the most orthodox clerics who saw in their ideas a strong Marxist bias. For this reason, the Theology of Liberation has generated great disputes, many of them still in force. In this paper we propose to return to one of these disputes by analyzing two texts that are key to understanding the theological movement and the controversies that arose within the Catholic Church. The work will be developed from a reading of the Concluding Document of the Second Conference of the Latin American Episcopate (1968) and of the Instruction on some aspects of the "Theology of Liberation" (1984) by Cardinal Joseph Ratzinger, who tried to warn about the possible "deviations" present in Liberation Theology.

Key words: Theology - Liberation - Vatican - Marxism - Latinamerica. 


\section{Cómo citar este artículo:}

APA: González, D. M. (2021). Teología de la Liberación: influencias, características y las tensiones con el Vaticano. Acheronta, №6, 50-75. Recuperado de: (agregar dirección web)

\section{La Teología de la Liberación: su caracterización y sus comienzos.}

La Teología de la Liberación tiene como momento de surgimiento la década de los 60'. Este movimiento tomó una gran relevancia en sus inicios y fue especialmente importante en los años 70' y 80. Esto debido a las posturas que defienden sus representantes y adeptos, pero sobre todo por el compromiso pastoral y político en la vida social de los países latinoamericanos. Para una breve caracterización del mismo, nos servimos de los aportes realizados por Juan Carlos Scannone en La Teología de la Liberación: Caracterización, Corrientes y Etapas, publicado en 1982 y un libro dedicado a la historia de la Teología de la Liberación, llamado Teología de la Liberación: los hechos esenciales en torno al movimiento revolucionario en América Latina, escrito por Philip Berryman y publicado en 1969. Además, nos serviremos de las lecturas del libro de Gutiérrez, su Teología de la Liberación: Perspectivas, publicado por primera vez en 1972.

Scannone (1982) define a la Teología de la Liberación como:

un movimiento teológico que ha marcado fuertemente la vida de la Iglesia y de la teología latinoamericanas, y que -nacido de la toma de conciencia eclesial de la situación injusta y de la correspondiente opción preferencial de la Iglesia por los pobres- ha contribuido y contribuye a fundamentarlas, ahondarlas y ampliarlas teológicamente. (p.11)

Esta toma de conciencia de la que habla el autor, tiene como veremos, varios antecedentes tanto teóricos y materiales que han propiciado el surgimiento de la Teología de la Liberación y sobre todo marcado su devenir. Mencionamos solo algunos de ellos. La toma de conciencia es en primer lugar, la conciencia de la pobreza que atraviesa el pueblo latinoamericano. El estado de miseria de la población campesina y 
obrera contrasta fuertemente con el poder y el lujo que detenta un sector pudiente, pero minoritario de la sociedad. Se trata entonces de la conciencia de una situación de injusticia y desigualdad social, que caló hondo en la reflexión de algunos teólogos, obispos, sacerdotes, y también laicos referentes sobre todo de comunidades de base; quienes en presencia de dicha situación se cuestionaron qué rol le cabía a la Iglesia en esa sociedad.

En cuanto al plano de las ideas influyeron en el movimiento y al contexto más general, hay quienes hablan de un cierto espíritu de la década que estaba signado por la simpatía más o menos generalizadas a ideas pro-revolucionarias. La revolución cubana (1959) y los movimientos de liberación nacional en el Tercer Mundo, las tensiones abiertas por la guerra fría y las revueltas juveniles en todo el mundo, cuya expresión más conocida es el mayo del 68 , son solo algunos de los aspectos que afectan a América y Europa en los años en curso.

Con una vocación fuertemente histórica y política, el movimiento que hoy llamamos Teología de la Liberación tiene un antecedente fundamental y que reúne en gran medida las posturas que después se expresarán y se diversificarán -ya sea por su radicalización o por su matización- en otros exponentes y participantes de la agrupación. El evento del que hablamos es la Conferencia Episcopal de Medellín en 1968. En esta Conferencia tienen lugar, por primera vez de forma sistematizada y condensada, las ideas que contribuyeron al desarrollo de la Teología de la Liberación y que darán lugar a la obra Teología de la Liberación. Perspectivas de Gustavo Gutiérrez; una de sus más logradas expresiones teóricas.

Gran parte de las ideas que posteriormente expondrán las diversas corrientes de la Teología de la liberación encuentran su primera formulación allí. Éstas, como bien dirá Scannone, dieron lugar a "un fecundo pero polémico replanteo global de la teología, lo que llevó a polarizaciones, ambigüedades, malentendidos y conflictos" (Scannone, 1982, p.11).

En este breve escrito nos proponemos hacer una comparación crítica en torno a la polémica que se suscitó en el interior de la Iglesia Católica a partir de la novedad 
Abril 2021.

que supuso la Teología de la Liberación para América Latina y para el mundo. Esta contraposición la haremos principalmente mediante una lectura del Documento Conclusivo de la Segunda Conferencia del Episcopado Latinoamericano, por las razones que arriba esgrimimos, y de la Instrucción sobre algunos aspectos de la "Teología de la Liberación" que fue firmado por Joseph Ratzinger en un intento por advertir sobre las posibles desviaciones que originarían las posturas de este movimiento teológico.

\section{Un primer momento: Los signos de la historia, la opción por los oprimidos}

En la Segunda Conferencia General del Episcopado Latinoamericano ${ }^{1}$ (SCGEL), celebrada en 1968 , se desarrollan una serie de discusiones y conferencias dictadas por los arzobispos, obispos y otros miembros importantes de la Iglesia en Latinoamérica. Todos ellos, siendo conscientes de que "para conocer a Dios es necesario conocer al hombre" (SCGEL, 1968 p.1) se proponen atender especialmente la realidad del hombre latinoamericano, su proceso histórico y la tarea que le cabe a la Iglesia en ese proceso. En este sentido, parten de considerar que:

La evangelización debe estar en relación con los signos de los tiempos. No puede ser atemporal, ni ahistórica. En efecto, los "signos de los tiempos" que en nuestro continente se expresan sobre todo en el orden social, constituyen un "lugar teológico" e interpelaciones de Dios. (SCGEL, 1968, p.26)

América Latina será entonces este lugar teológico, que orientará tanto las conclusiones de la Conferencia como las propuestas futuras de la Teología de la Liberación. Como dijimos, las ideas y conclusiones a las que llegan parten de un análisis del proceso histórico de Latinoamérica. La responsabilidad de la Iglesia en ese proceso es asumida también como una responsabilidad de su rol tanto en la conquista y la colonización como en los siglos siguientes, sobre todo en las luchas de los pueblos latinoamericanos por su independencia. Así en el documento se puede leer que la

\footnotetext{
${ }^{1}$ De ahora en más SCGEL o Conferencia de Medellín.
} 
misma "Acata el juicio de la historia ${ }^{2}$ sobre esas luces y sombras, y quiere asumir plenamente la responsabilidad histórica que recae sobre ella en el presente" (SCGEL, 1968, p.1).

Un análisis interesante sobre algunos de estos puntos se los debemos a Enrique Dussel, quien ha brindado grandes aportes en sobre la historia de la Iglesia Católica y su rol en América Latina. La Iglesia formó parte, durante la conquista, de un proceso de colonización que tenía como modelo la sociedad occidental, europea y cristiana. Tanto la Corona como el poder eclesial compartían ese proyecto "civilizatorio". Una de las pocas excepciones es la de Bartolomé de las Casas, un sacerdote que en la época de la colonización tomó consciencia de crueldad a la que se vieron sometidos los "indios" en este proceso y reorientó su vida a la defensa de los mismos. Otro momento importante es la postura a favor de la Corona que adoptó la Iglesia en las revoluciones por la independencia.

Las rupturas con la Corona fueron impulsadas por las elites nacionales, a quienes motivaba especialmente la idea de libertad comercial y económica. Y el resto de la población, los hombres pobres, no sólo lucharon en las primeras líneas de batalla, sino que además no se vieron beneficiados en las dependencias obtenidas. Esto marcará también que la idea misma de independencia sea discutida, como lo veremos más adelante. Pese a ello, lo que es preciso destacar es el rol marcadamente conservador que ha adoptado la Iglesia cuando de disputas sociales, políticas y culturales se trataba.

Dicho esto, cobra importancia la pregunta que explora Phillip Berryman acerca de “¿Cómo es posible que de una Iglesia tan históricamente conservadora pueda surgir una teología de la liberación?" (Berryman, 1989, p.11). Las respuestas a este interrogante serán mayoritariamente históricas, tal como los hechos que ya mencionamos. Sin embargo, otro hecho -que además de histórico, tiene gran peso por su contenido teológico- es el Concilio Vaticano II abierto en 1962. Las discusiones y conclusiones que se esgrimieron allí propiciaron un impulso para considerar con más

\footnotetext{
${ }^{2}$ La cursiva es nuestra.
} 
Abril 2021.

seriedad y con un compromiso pastoral más activo la situación particular de América Latina. La fórmula guía del Episcopado reunido en Medellín fue «VER, JUZGAR, OBRAR»; y si bien la misma puede ser descriptiva del proyecto, una caracterización como la de Berryman puede ayudar a dimensionar la trascendencia que el Concilio II tuvo:
Antes del concilio a los católicos se les enseñaba que su principal deber en la vida era permanecer en "estado de gracia" y alcanzar el cielo. La Iglesia era la mediadora de la gracia y la verdad. En semejante esquema los asuntos terrenales eran finalmente insignificantes. En el Vaticano II (...) la Iglesia católica aceptó modestamente su condición de "peregrina" que camina al lado del resto de la humanidad. En un posterior giro radical, la Iglesia empezó a considerar al "progreso humano" como evidencia de la labor de Dios en la historia humana. (Berryman, 1989, p.13)

Este posterior giro radical al que se refiere Berryman es justamente el que inicia la SCGEL y que se profundiza con el desarrollo de la Teología de la Liberación. En esta línea, es interesante remarcar la expresión sobre el juicio de la historia, el peso que se le da a la dimensión histórica es una constante en las diversas expresiones de la Teología de la Liberación ${ }^{3}$. Precisamente, este será uno de los aspectos más criticados en la Instrucción Sobre Algunos Aspectos De La "Teología De La Liberación"4 de Ratzinger, como veremos más adelante. Se presenta luego del Concilio y en particular en la Conferencia de Medellín una toma de conciencia del presente del hombre latinoamericano, un análisis del pasado y las primeras consideraciones sobre los cambios que serán necesarios para un futuro favorable. Se mencionan y se consideran especialmente la situación de aquellos sectores oprimidos, altamente desfavorecidos por el subdesarrollo de los países latinoamericanos, pero también por la dependencia a la estos que están sujetos. Entre los sectores más perjudicados se encuentran los

\footnotetext{
${ }^{3}$ En Teología de la Liberación: perspectivas, Gutiérrez (1975) acentuará el papel del sujeto de la historia como un elemento clave para comprender su actualidad: "Es el comportamiento de un hombre cada vez más consciente de ser sujeto activo de la historia, cada vez más lúcido frente a la injusticia social y a todo elemento represivo que le impida realizarse, cada vez más decidido a participar en la transformación de las actuales estructuras sociales y en la efectiva gestión política" (pp.75-76)

${ }^{4}$ De ahora en más Instrucción.
} 
campesinos, artesanos e industriales, las mujeres que reclaman tratos y derechos igualitarios, los jóvenes, la clase media, profesionales que no encuentran lugar para el desarrollo de su profesión por el subdesarrollo que caracteriza a sus países natales, etc. La descripción de la situación que atraviesa Latinoamérica es significativa, se hablará de que "Una miseria, como hecho colectivo", es una injusticia que clama al Cielo" (SCGEL, 1968, p.2).

Este punto de partida tiene resonancias importantes a la hora de pensar la actividad teológica, ya que, podríamos decir, determina la forma en la que la Teología es entendida en su proceder. En otras palabras, diríamos que invierte el procedimiento seguido por la Teología Tradicional. La nota es metodológica, y por lo mismo no carece de importancia. La Teología, la Iglesia y la pastoral serán pensadas desde y posteriormente al análisis de la realidad latinoamericana. La realidad del o los pueblos latinoamericanos es la que tiene preeminencia, aparece antes en la consideración y por tanto, demarca el camino a seguir. Dirá Berryman (1989) que “El procedimiento mismo -empezar con observaciones sobre la sociedad y después considerar a la Iglesia - era un rompimiento con el método tradicional de la doctrina-a-la aplicación que insinúa que la verdad llega a la tierra de lo alto" (p.18). Las consideraciones respecto de la Iglesia y su inserción social, y de la Teología serán posteriores y su evaluación se hará a la luz de la realidad latinoamericana.

Expuestos estos puntos de partida, los teólogos latinoamericanos empiezan a discutir y a proyectar maneras novedosas de entender y afianzar una praxis de la cristiandad, desde la perspectiva única y particular que supone el contexto latinoamericano; expresión de ello es la manera en que inicia el discurso:

En esta transformación detrás de la cual se expresa el anhelo de integrar toda la escala de valores temporales en la visión global de la fe cristiana, tomamos consciencia de la "vocación original" de América Latina: vocación de aunar en una síntesis nueva y genial, lo antiguo y lo moderno, lo espiritual y lo temporal, lo que nosotros nos entregaron y nuestra propia originalidad. (SCGEL, 1968, p.2)

\footnotetext{
${ }^{5}$ La cursiva es nuestra.
} 
En lo que sigue veremos cómo se interpreta doctrinalmente esta vocación cristiana en América Latina, desde la perspectiva que inaugura la Conferencia de Medellín. Así como también las reacciones que desde el Vaticano se generaron por la gran difusión y polémicas en torno a este movimiento.

Hacia una apertura epistemológica: la situación de pecado y el problema de la unidad de la historia

Como dijimos anteriormente, el Episcopado reunido en Medellín fija su punto de partida: la situación de justicia y de miseria que vive gran parte del pueblo Latinoamericano. Las posturas que adoptan al respecto surgen de la necesidad de comprender esta situación como parte de un problema estructural. Esta visión colectiva acerca de una problemática que se considera estructural es lo que conlleva también a que las soluciones propuestas adquieran este matiz fuertemente colectivista. Las lecturas e interpretaciones también estarán determinadas por el deseo de promover una praxis cristiana concordante con estos sentidos. En este trabajo insistiremos en una de las consecuencias más inmediatas que suscita este enfoque: la de otorgar mayor consideración a la praxis pastoral en la transformación política y social, en desmedro de lo que se podría considerar como la tarea más tradicional y doctrinal de evangelización que defiende la Iglesia.

La comprensión de los problemas desde una perspectiva estructural, encontrará su primer eco teológico en la concepción acerca del pecado. En determinado momento, se llama la atención sobre la cuestión del pecado, y si bien se expresa que toda transformación debe partir primero desde la interioridad de los hombres, algunos apartados parecen indicar otra lectura. El párrafo siguiente llama especialmente la atención:

Al hablar de una situación de injusticia nos referimos a aquellas realidades que expresan una situación de pecado; esto no significa desconocer que, a veces, la miseria en nuestros países puede tener causas naturales difíciles de superar. (SCGEL, 1968,

p.7) 
La referencia a una situación de pecado es particularmente llamativa. Desde esta perspectiva, el párrafo puede indicar que ciertos hechos o acontecimientos en los países latinoamericanos indicarían, paradójicamente, la presencia de una falta o pecado. El poner el acento en el estado de la situación y no en los sujetos pecadores individualizados, hace posible pensar que la tesis de la transformación interior es insuficiente para cambiar esa realidad; es necesario un cambio de las estructuras mismas, ya sea, económicas, políticas o sociales.

Sobre este último punto también se erigirá la crítica que se emite desde el Vaticano, y su "llamado de atención" ante posibles desviaciones en las tareas, que se entienden, le corresponden a la Iglesia. Veremos esto en el próximo apartado al tratar lo que desde teología tradicional se percibe como una inversión de prioridades; o mejor dicho, en lo que se dominará el pasaje de la ortodoxia a una ortopraxis.

A pesar de que en los anuncios de la Conferencia de Medellín se intenta aunar los esfuerzos de una praxis liberadora que no deje de lado la tarea Evangelizadora por medio de la Palabra, los primeros signos de esta "inversión" se muestran desde principio. Por ejemplo cuando se expresa que "No baste por cierto reflexionar, lograr mayor clarividencia y hablar; es menester obrar. No ha dejado de ser esta la hora de la palabra, pero se ha tornado, con dramática urgencia, la hora de la acción" (SCGEL, 1968, p.1).

Este deseo de motivar en La Iglesia en su conjunto a una mayor presencia y participación activa en los procesos de transformación social, encuentra, según lo entiende el Episcopado Latinoamericano, sus fundamentos teológicos en la Palabra Escrita. De allí que se propone una nueva Teología, que tenga como motor la liberación de los pueblos latinoamericanos y la promoción humana. Se trata de la aplicación de lo que se comenzó a gestar en el Concilio Vaticano II bajo el lema "VER, JUZGAR, OBRAR". La novedad, sin embargo, está dada por el hecho de que su aplicación se hace desde, en y para el pueblo de América Latina.

Se tratará entonces de asumir una Teología que interprete la situación particular de América Latina como un llamamiento o signo histórico para una praxis 
Abril 2021.

cristiana, de promoción humana y de transformación social. En este punto, en la conferencia se puede observar una postura de considerable apertura epistemológica, sustentada en la idea de que se han realizado en las ciencias sociales y humanas grandes esfuerzos y avances teóricos que permiten comprender con mayor suficiencia la realidad social de América Latina.

Estas fuentes de conocimientos serán tenidas en cuenta en uno de los niveles propios de la práctica teológica, que Scannone llamará el nivel científico, en el cual:

La Teología de la Liberación usará no solo la mediación de la conceptualidad teológica (que a su vez supone la filosófica), sino también la de las ciencias históricas y sociales, para conocer científicamente la situación histórica y para una crítica científica de las ideologías que impiden este conocimiento. (Scannone, 1982, p.4)

Dicho esto, debemos señalar que en esta Conferencia el Episcopado intenta asumir una posición crítica acerca de los aportes de ciertas teorías sobre lo social. Por ello, se detienen a denunciar tanto los perjuicios del sistema liberal capitalista como del marxismo; ya que sostienen que se trata de dos tendencias perjudiciales para el pueblo Latinoamericano.

Dicho esto, en el diagnóstico acerca de la situación que viven los países de Latinoamérica, tanto los conceptos y el marco teórico utilizado, dan cuenta de una gran influencia marxista, además de lecturas concordantes con perspectivas propias de las Teorías de la dependencia. Para dar cuenta de la presencia de estos marcos teóricos en el diagnóstico y los análisis que hace el Episcopado latinoamericano, mencionaremos algunos de los aspectos nodales de la Conferencia de Medellín, especialmente en los apartados orientados a ser una exposición de la situación de la región.

Todos los ítems que se reúnen bajo el subtítulo "La Justicia" dan cuenta de una lectura compleja de la situación de América Latina, además en el subtítulo denominado "La Paz", se caracteriza la situación de América Latina de tal forma que refleja la influencia de ciertos marcos de análisis propios de las ciencias sociales y la filosofía. Así por ejemplo en el ítem “La situación Latinoamericana y la Paz" se 
menciona que "Si "el desarrollo es el nuevo nombre de la paz" el subdesarrollo Latinoamericano, es una injusta situación promotora de tensiones que conspiran contra la paz" (SCGEL, 1968, p.7). En la Conferencia de Medellín, esta distorsión de la paz en Latinoamérica se analiza a partir de tres grandes nociones problemáticas, la primera denominada "Tensiones entre clases y colonialismo interno", la segunda llamada "Tensiones entre clases y neocolonialismo externo" y por último se menciona "Tensiones entre los países de América Latina".

Sobre las tensiones entre clases y colonialismo se dirá que existen “Desigualdades excesivas entre las clases sociales, especialmente, aunque no en forma exclusiva, en aquellos países que se caracterizan por un marcado clasicismo: pocos tienen mucho <cultura, riqueza, poder, prestigio> mientras muchos tienen poco" (SCGEL, 1968, p.7). Por el momento, nos interesa destacar en este análisis la utilización y la adopción conceptos como el de "clases" para comprender la dinámica social de Latinoamérica. En parte, la adopción de estos enfoques determinará también la necesidad de una toma de postura hacia la promoción de los sectores oprimidos.

Esta toma de posición ya marca de alguna manera lo que serán algunos los aspectos principales de los teólogos de la liberación, nos referimos particularmente al caso de Gutierrez cuya teología se funda en idea de una "opción por los pobres". Cabe decir, que Guitierrez participó activamente de la SCGEL y que además fue uno de sus principales expositores. Sus ideas, se ven claramente reflejadas en el documento que analizamos aquí. Así, por ejemplo, se sostiene como tarea pastoral:

Defender, según el mandato evangélico, los derechos de los pobres y oprimidos, urgiendo a nuestros gobiernos y clases dirigentes para que eliminen todo cuanto destruya la paz social: injusticias, inercia, venalidad, insensibilidad; Denunciar enérgicamente los abusos y las injustas consecuencias de las desigualdades excesivas entre ricos y pobres, entre poderosos y débiles, favoreciendo la integración. (SCGEL, 1968, p.11)

La defensa de los sectores oprimidos y menos favorecidos conlleva también a la crítica de quienes no se encuentran en esta situación mientras que de los sectores 
Abril 2021.

ISSN 2344-9934

dominantes se dirá que "califican de acción subversiva todo intento de cambiar un sistema social que favorece la permanencia de sus privilegios" (SCGEL, La Paz, 1, P4).

\section{En cuanto a las tensiones internacionales y neocolonialismo externo,} observamos una lectura concorde a las Teorías de la Dependencia ${ }^{6}$, ya que no solo se habla de un subdesarrollo sino también de "las consecuencias que entraña para nuestros países su dependencia de un centro de poder económico (...) nuestras naciones con frecuencia no son dueñas de sus bienes ni de sus decisiones económicas" (SCGEL, 1968, p.8). Estos aspectos son relevantes, las teorías tradicionales a las que los marcos teóricos de la teoría de la dependencia van a criticar, no solo sostienen que la situación de América Latina debe leerse en términos de progreso, lo que sugiere que la región estaría situada en un estadio aún no desarrollado. Esta lectura indica además, que el camino a seguir sería el de la imitación o la adopción de los países atrasados (no desarrollados) de las recetas utilizadas por los que se encuentran en este estadío más "avanzado" (desarrollado). Por ello, estas lecturas no sólo diagnostican desde afuera el problema económico de Latinoamérica, sino que sugieren la solución; a saber, imitar a los países desarrollados.

Se destaca además la evidente interdependencia del ámbito económico y político. Y la fuerte importancia de que a un desarrollo económico también le sobrevenga una autonomía en las decisiones propias de cada nación, de ello se desprende la denuncia de que los "principales culpables de la dependencia económica de nuestros países son aquellas fuerzas que, inspiradas en el lucro sin freno, conducen a la dictadura económica y al <imperialismo internacional del dinero>" (SCGEL, 1968, p.8).

\footnotetext{
${ }^{6}$ Recordemos que las Teorías de la Dependencia bregan por una lectura integrada según la cual la situación de algunos países no se puede comprender solo por la división desarrollo-subdesarrollo, sino por las relaciones de centro-periferia. "La situación de subdesarrollo se produjo históricamente cuando la expansión del capitalismo comercial y luego del capitalismo industrial vinculó a un mismo mercado economías que, además de presentar grados diversos de diferenciación del sistema productivo, pasaron a ocupar posiciones distintas en la estructura global del sistema capitalista. De ahí que entre las economías desarrolladas y las subdesarrolladas no sólo exista una simple diferencia de etapa o de estado del sistema productivo, sino también de función o posición dentro de una misma estructura económica internacional de producción y distribución. Ello supone, por otro lado, una estructura definida de relaciones de dominación." (Cardoso y Falleto, p.12)
} 
Todo este diagnóstico, con una marcada influencia terminológica de las Ciencias Sociales será el gran foco de cuestionamiento por parte del Vaticano, como veremos a continuación. Esto se debe en gran parte a que la adopción de ciertos marcos teóricos conlleva una revisión teológica que tendrá graves consecuencias doctrinales en aspectos muy arraigados en el seno de la tradición teológica Occidental. Una de las consecuencias, es la inminente ruptura de una visión dualista de la historia que sostiene una historia terrenal y una espiritual que finalmente determinará el fin de los tiempos históricos.

Los primeros puntos de ruptura con esta visión serán expuestos en la Conferencia. Por ejemplo, cuando se menciona que "En la búsqueda de la salvación debemos evitar el dualismo que separa las tareas temporales de la santificación" (SCGEL, 1968, p.3). Si bien de nuevo se insiste en que no se trata de identificar el Reino de Dios con el orden temporal mundano, el punto de quiebre está en expresiones tales como:

Cristo, activamente presente en nuestra historia, anticipa su gesto escatológico no sólo en el anhelo impaciente del hombre por su total redención, sino también en aquellas conquistas, que como signos pronosticadores, va logrando el hombre a través de una actividad realizada en el amor. (SCGEL, 1968, p.1)

Esta insistencia en la promoción humana a nivel material será muy significativa y característica de la Teología de la Liberación. Asimismo, la adopción de un marco de análisis de las Ciencias Sociales, la tendencia a la unidad de la historia, la opción por los oprimidos o los pobres como una elección pensada a partir del concepto de clases y la mención a un cierto anticipo escatológico constituirán algunos de los elementos que mayor polémica susciten al interior de la doctrina católica y que serán puestos en cuestión en la instrucción papal.

Una vuelta a la ortodoxia: el llamado a una crítica epistemológica, la evangelización universal y la Trascendencia del Reino de los Cielos. 
El 6 de agosto de 1984 el Vaticano, emite un documento firmado por Joseph Ratzinger que, con la aprobación de Juan Pablo II, pretende ser un llamado de atención hacia las posturas defendidas bajo diversas corrientes de pensamiento que podrían reunirse en la categoría de Teologías de la Liberación. Es importante señalar además, que esta mención de las Teologías de la Liberación en plural, la realiza Ratziger, no así lo teólogos de la liberación, como bien lo nota Berryman?

Si bien el escrito expresa que "Esta llamada de atención de ninguna manera debe interpretarse como una desautorización de todos aquellos que quieren responder generosamente y con auténtico espíritu evangélico a <la opción preferencial por los pobres>" (Ratzinger, 1984, p.262), en el desarrollo de la exposición se acentuará cada vez más su reprobación a diversas posturas que forman parte de la Teología de la Liberación. Intentaremos mostrar esta crítica en sus diversos elementos centrales.

En el documento se lee lo siguiente: "algunos se sienten tentados a poner el acento de modo unilateral sobre la liberación de las esclavitudes de orden terrenal y temporal, de tal manera que parecen hacer pasar a un segundo plano la liberación del pecado." (Ratzinger, 1984, p.262). El primer punto que se acentúa entonces, es insistir en que para la doctrina cristiana el hecho de que las esclavitudes terrenales existan solo puede deberse a la existencia originaria del pecado en el hombre; por ello "La primera liberación, a la que han de hacer referencia todas las otras, es la del pecado" (Ratzinger, 1984 p.267). Lo que indica además que la tarea de la evangelización deberá poner la atención en la liberación del pecado, verdadera causa de toda otra falta. Es al nivel de la consciencia y del espíritu, según entiende Ratzinger, hacia dónde debería dirigirse el compromiso evangelizador.

\footnotetext{
${ }^{7}$ Al respecto dirá: “Esta estratagema, que inició el obispo López Trujillo a principios de los setenta, implica que hay variedades aceptables e inaceptables de teología de la liberación. Ya que no se mencionan nombres, es imposible determinar cuál es verdaderamente considerada como aceptable. Los mismos teólogos - Gutiérrez, Segundo, Dussel, Sobrino, los Boff, Assmann, Ellacuría, Vidales, Comblin, Richard y Muñoz - pueden diferir en estilo y en enfoque, y pueden no estar de acuerdo en algunos asuntos, pero se refieren a la teología de la liberación en singular, ya que ven que sus propios esfuerzos apoyan lo que es esencialmente un proceso histórico simple" (Berryman, 1989, p.133)
} 
Por otro lado, si bien se anuncia como una necesidad de la Iglesia el promover la igualdad y bienestar de todos los hombres, se denuncia que esto no debe confundir la tarea propia que le corresponde. El punto de partida que más arriba denominamos como el lugar desde el cual se piensa y se gesta la Teología de la Liberación, es, en las instrucciones del Cardenal, puesto en un segundo plano. Con respecto a ello se expresa que "No se puede tampoco localizar el mal principal y únicamente en las estructuras económicas, sociales o políticas malas" (Ratzinger, 1984, p.268). La idea o al menos la expresión que destacamos antes de una situación de pecado, queda así desestimada totalmente.

El contra-argumento del Cardenal sostiene que: "La raíz del mal, reside pues, en las personas libres y responsables, que deben ser convertidas por la gracia de Jesucristo" (Ratzinger, 1984, p.268). Insistimos en estos pasajes ya que sugieren el primer indicio de lo que será la llamada a una vuelta a la ortodoxia; mediante la insistencia en que lo defectuoso de la Teología de la Liberación es el haber invertido el orden de prioridades, dirigiéndose más a una transformación social que a la causa que genera un orden social injusto: el pecado.

El segundo punto será el de señalar que para algunos- entiéndase, los teólogos de la liberación- "la lucha necesaria por la justicia y la libertad humanas, entendidas en su sentido económico y político, constituyen lo esencial y el todo de la salvación. Para estos, el Evangelio se reduce a un evangelio puramente terrestre" (Ratzinger, 1984, p.270). Este evangelio puramente terrenal concluye, según el Cardenal, en la idea de que lo más importante es un cambio de estructuras y no una transformación espiritual. Es interesante remarcar al respecto, que los Teólogos de la Liberación entienden este cambio social, económico y político como urgente, no como único y fundamental.

Ratzinger -en su rol de jefe de la Sagrada Congregación para la doctrina de la feno puede más que interpretar esta tendencia a priorizar el aspecto terrenal por sobre el espiritual como una desviación que será necesaria atender, guiar e iluminar para una correcta interpretación del Mensaje Bíblico. Recordemos, que en la Conferencia de Medellín los obispos se cuidaron de aclararlo, expresando que: "No confundimos el 
Abril 2021.

ISSN 2344-9934

progreso temporal y el Reino de Cristo" (SCGEL, 1968, p.3). Sin embargo, la mención a la anticipación escatológica y la gran preocupación por la interpretación de los signos de los tiempos históricos, así como también la explícita opción por los pobres y las duras críticas a las elites nacionales y clases dirigentes son las que recorren todo el documento y las que a los ojos del Vaticano resonará con mayor fuerza.

Ante ello, Ratzinger manda un mensaje de atención. El Cardenal no solo se propone otras interpretaciones de algunos pasajes de la Biblia sino que concentra todos sus esfuerzos en explicar cómo fue posible que tal interpretación haya sido concebida por los obispos latinoamericanos. El origen del error lo encontrará, en lo que entiende, es una adopción acrítica por parte de la Teología de la Liberación de ciertos instrumentos del pensamiento que no son conciliables con la doctrina teológica de la Iglesia. Más directamente, el marxismo será el gran responsable de esta mala lectura inicial y de las desviaciones siguientes.

En las instrucciones del Cardenal se insiste en la necesidad de una crítica epistemológica que actúe de filtro a fin de notar cuáles principios o herramientas propios de otros campos del saber no pueden ser aceptados por la doctrina cristiana. En referencia a ello se recomendará que "la utilización por la teología de aportes filosóficos o de las ciencias humanas tiene un valor instrumental y debe ser objeto de un discernimiento crítico de naturaleza teológica" (Ratzinger, 1984, p.273). En este sentido, se incitará a evaluar todos aportes de las ciencias sociales a la luz de la fe y la Verdad Revelada, lo que da lugar a un desarrollo que acentúa cada vez más la diferencia entre el estatus de "Verdad" de la Palabra Revelada y el status de aporte meramente "conjetural" de las diversas disciplinas abocadas al estudio de lo social.

Como veremos, el principal blanco de ataque será el haber adoptado la terminología y los marcos de análisis provenientes del marxismo. Scannone también señala esta influencia y destaca que una de las corrientes de la Teología de la Liberación es la más abierta a esta utilización, la que él llama Teología desde la praxis de grupos revolucionarios. Según la postura de esta corriente sería posible distinguir un nivel de análisis que permiten los aportes marxistas y un nivel ideológico que la 
teología desecharía, por ello "para comprender la realidad usa el análisis marxista (materialismo histórico) con pocos correctivos, aunque no acepta el materialismo dialéctico, que es ateo" (Scannone, 1982, p.6).

Si seguimos lo mencionado por Scannone, sí se puede observar la aplicación de un examen crítico orientado a la revisión de los planteos marxistas para dejar de lado aquello que no concuerda con la doctrina cristiana de la Iglesia. Sin embargo, desde lo expuesto por la Instrucción, esta aceptación parcial de los postulados marxistas tampoco sería aceptable a la luz de la enseñanza doctrinal oficial. Ratzinger afirma que para aceptar los postulados marxistas es necesario antes haber aceptado unos a priori ideológicos que sustentan la teoría. Finalmente, la aceptación de unos postulados (materialismo histórico) tendrá como consecuencia la aceptación de la ideología (materialismo dialéctico). Citamos en extensión el argumento 2 del capítulo Subversión del Sentido de la Verdad y Violencia:

En la lógica del pensamiento marxista, el "análisis" no es separable de la praxis y de la concepción de la historia a la cual está unida esta praxis. El análisis es así un instrumento de crítica, y la crítica no es más que un momento de combate revolucionario. Este combate es el de la clase del Proletariado investido de su misión histórica. (Ratzinger, 1984, p.274)

Lo anterior expresa un encadenamiento sencillo y no por ello, menos importante en la crítica a la Teología de la Liberación. Lo que indicaba Scannone y que también podemos ver en las posturas de algunos teólogos de la liberación es el uso de ciertos conceptos que se toman como herramientas útiles para comprender la realidad latinoamericana. Y si bien el marco conceptual es más amplio, la mayor preocupación del Vaticano es la utilización de conceptos marxistas.

Según lo entiende Ratzinger el error que desencadenó todas las desviaciones con respecto a la ortodoxia es la creencia ingenua en que la aceptación de ciertos marcos de análisis, no incluía la aceptación de toda la "ideología marxista". La instrucción señala que admitir la dialéctica de clases, conlleva necesariamente a admitir una visión determinada de la historia, la de la lucha de clases y la necesaria 
Abril 2021.

ISSN 2344-9934

revolución. Y es por tanto también, aceptar el rol que a cada uno le compete en esa lucha. La Teología de la Liberación, en su opción preferencial por los pobres, habría aceptado este hecho, a su vez tomó partido y por ende, dejó de lado una doctrina eclesial fundamental, la de la evangelización universal.

Es necesario que rescatemos al menos tres finalidades en este análisis crítico que propone la Instrucción. La primera es acentuar el carácter marcadamente político que afecta a la Teoría de la Liberación, algunas expresiones de Guitierrez (1975) son relevantes al respecto "La razón humana se ha hecho razón política" (p.76) dirá. Esto es interpretado por la sede papal como la prueba de que la preocupación terrenal ha ganado terreno por sobre la preocupación espiritual y la evangelización. El segundo y no menos importante es el señalar que se ha comenzado a adoptar una posición partidaria, que en La Conferencia de Medellín estaba identificada con la figura de los oprimidos y los pobres de América Latina y en posturas como la de Guitierrez se relaciona cada vez con más fuerza a la última de ellas.

Por tanto, se denuncia que esta conciencia es partidaria y se aleja mucho del mensaje cristiano que postula "el mandamiento de amor fraterno extendido a todos los hombres (...) No hay discriminaciones o límites que puedan oponerse al reconocimiento de todo hombre como el prójimo" (Ratzinger, 1984, p.267). Se entiende que se busque una vuelta a un mensaje menos politizado de los teólogos latinoamericanos, quienes adoptaron posturas muy críticas con las elites naciones y los dirigentes de los países latinoamericanos.

Dicho esto, se entiende que el haberse optado partidariamente por un sector social es producto de haber aceptado previamente análisis sociológicos en términos de clases. A los que se opone una clase dominante que se debe compartir. Esto implica además, aceptar postulados acerca de la dinámica social y los procesos por los cuales esa dinámica de lucha entre opresores-oprimidos habrá de llegar a su fin. En el capítulo anterior mostramos esta tendencia a combatir una dualidad de la historia, mediante la idea de una anticipación de la historia. La crítica que lanza la Instrucción es clara: 
Se afirmará que Dios se hace historia, se añadirá que no hay más que una sola historia en la cual no hay que distinguir ya entre historia de la salvación e historia cristiana.

Mantener esta distinción sería caer en el dualismo. (Ratzinger, 1984, p.276)

La tarea final será acentuar la trascendencia del Reino de los Cielos. Insistir en que la imposibilidad de identificar a este último con el mundo terrenal y por ende hacer un llamamiento a promover la verdadera causa del mensaje que es la evangelización de los hombres. La verdadera y auténtica liberación será entonces la liberación final del pecado, imposible de anticipar terrenalmente.

\section{La ideología y la violencia ¿Obstáculos insuperables?}

Finalmente nos gustaría mencionar dos aspectos que consideramos son los obstáculos, tal vez insalvables, que originan la polémica entre la Teología de la Liberación y la respuesta del Vaticano. Nos referimos específicamente a la cuestión de cómo se piensa, de un lado y del otro, la ideología y la violencia.

En cuanto a la ideología, nos permitimos citar la diferenciación que hace Berryman de acuerdo a lo que establecieron algunos teólogos Latinoamericanos, que en vísperas de la Conferencia de Puebla, se reunieron en 1978 en Caracas y clasificaron tres maneras de comprender la ideología. En un primer sentido, propio del marxismo, se entiende a la ideología como la imposición de una cierta manera de ver el mundo, que se ejerce de una clase dominante a otra clase, generalmente oprimida; es una falsa consciencia. En un segundo sentido, la ideología implica una manera de entender el mundo de manera totalizante, es una suerte de cosmovisión que lo abarca todo y pretende explicar la realidad. Por último, "denota «un sistema de medios y fines para confrontar un período particular de la historia en sus circunstancias diferentes y cambiantes y para dirigir a la historia hacia fines que son parciales y que están sujetos a revisión»" (Berryman, 1989, p. 94).

Según esta descripción, los teólogos de la liberación aceptan el tercero de estos sentidos como propio. Es decir, confirman su propia implicación en una manera de 
entender la ideología. Esta manera de entenderla supone varias cuestiones que son un interés teórico y práctico muy importante. En un sentido, se acepta que las posturas parten de una determinada toma de posición, se acepta su carácter político, ya que se trata de medios de participación política y con fuerza histórica. Y por lo mismo, se acepta la posibilidad, siempre abierta, de la falibilidad de esas ideas y medios.

Por otro lado, las posturas más ortodoxas, parecen estar atravesadas aún por la idea de infalibilidad. La doctrina de la Iglesia sería así, aquella verdad, no sujeta a mucha discusión ni revisión. Esto es así, porque en parte, está unida a la idea de infalibilidad, la idea de la neutralidad e imparcialidad acerca de cómo se conciben los "asuntos terrenales".

Cabría preguntarse entonces, si este intento de separar tajantemente los asuntos religiosos y políticos es de hecho posible. ¿Puede la iglesia, como institución, ser neutral a los asuntos políticos de su tiempo? No hace falta demasiada revisión para confirmar que la Iglesia ha estado fuertemente unida a la política, tal como lo muestran los antecedentes que antes citamos acerca del rol eclesial en la conquista y la colonización, por nombrar solo una de sus vinculaciones. El lector podrá encontrar muchos ejemplos más, en donde el poder eclesial y político aunaron fuerzas, no siempre con los fines más genuinos.

Por otro lado, la Teología de la Liberación pone en duda y niega la posibilidad de ser neutral y de separar tajantemente los planos religiosos y políticos. El problema más relevante al respecto, no es ideología sí o ideología no, al menos si entendemos ideología en este tercer sentido. La cuestión es si se acepta que se tiene una ideología, o que uno se encuentra influenciado por elementos ideológicos o se niega tal influencia. La ortodoxia, parece inclinarse en este último sentido; negando en el plano formal sus influencias y en el plano político su injerencia. En este sentido, compartimos la apreciación de Berryman (1989):

parece claro que cuando el papa y otros objetan la injerencia del clero en la política, su preocupación verdadera es un tipo particular de injerencia, aún cuando la objeción se expresa en términos de principios generales. (pp.91-92) 
Siguiendo la línea del autor, podríamos decir que la Teología de la Liberación pone en jaque esta pretensión de neutralidad, admitiendo que es imposible ponerse por encima de la ideología en un sentido general. En oposición a ello, las posturas de la ortodoxia aparece en su aspecto más profundamente ideológico, en el sentido en que busca ocultarse como ideología, asumiendo para sí el lugar de la verdad. La ideología de los teólogos tradicionales se presenta bajo la figura la verdad, la neutralidad, religiosidad "pura" o apartidaria, más allá de toda ideología, y es justamente en ese ocultamiento donde se expresa su carácter ideológico. El discurso de Ratzinger, por muy ortodoxo que se presente, no puede desligarse de los elementos propios de su tiempo; su crítica al marxismo está atravesada por su experiencia particular, la experiencia europea del socialismo soviético. Cuando Raztinger (1984) menciona que:

Un hecho notable de nuestra época debe ser objeto de la reflexión de todos aquellos que quieren sinceramente la verdadera liberación de sus hermanos. Millones de nuestros contemporáneos aspiran legítimamente a recuperar las libertades fundamentales de las que han sido privados por regímenes totalitarios y ateos que se han apoderado del poder por caminos revolucionarios y violentos, precisamente en nombre de la liberación del pueblo. No se puede ignorar esta vergüenza de nuestro tiempo (...) Quienes se vuelven cómplices de semejantes esclavitudes, tal vez inconscientemente, traicionan a los pobres que intentan servir. ( p.283)

Lo que en realidad hace es asimilar la experiencia europea con la utilización de términos del marxismo por parte de los teólogos de la liberación. La extrapolación es exagerada no solo por la innegable diferencia entre un hecho y otro, sino también porque acusa de complicidad a los teólogos de la liberación y de inconsciencia a la hora de utilizar categorías de análisis propias de las ciencias sociales. Berryman es aún más tajante al respecto, expresa que "El tono algo condescendiente implica que los teólogos latinoamericanos necesitan una lección de historia de un europeo" (Berryman, 1989, p.138). Los ve incapaces de reflexionar desde la doctrina cristiana su propio tiempo y lugar. Esta calificación es mínimamente extremista; supone la falta de total sentido crítico en los representantes de la teología de la Liberación, así como 
Abril 2021.

ISSN 2344-9934

también eleva la realidad europea a criterio universal sobre el que se evaluarán todas las demás experiencias.

En cambio, desde la teología de la liberación la cuestión de la ideología tiene también su argumentación doctrinal. Se debe a la gran presencia de la cristología en los países latinoamericanos. La figura de Jesus, su vida y su obra han servido como mayor ejemplo teológico, por sobre otros aspectos más metafísicos. En este sentido, se trata de ver la vida de Jesús como un ejemplo desde el cual evaluar en perspectiva la propia praxis. Así

Juan Luis Segundo afirma explícitamente que Jesús, al compartir la condición humana, también adquirió los elementos ideológicos de su época, y están reflejados en el Nuevo Testamento. Para estar por encima de toda ideología uno tendría que estar por encima de la condición humana. (Berryman, 1989, p.138)

Uno de los aspectos, que nos servirá más bien como ejemplo de este atravesamiento ideológico, es la cuestión de la violencia. En América Latina se viven, en la experiencia de los teólogos de la liberación, momentos de suma violencia. Muchas veces, esta violencia es caracterizada como violencia institucionalizada. Su diagnóstico es que: "La opresión ejercida por los grupos de poder puede dar la impresión de mantener la paz y el orden, pero en realidad no es sino "el germen continuo e inevitable de rebeliones y guerras" (SCGEL, 1968, p.9). Los Estados latinoamericanos, dirigidos por elites que se encuentran muy alejadas de la realidad del pueblo trabajador, no brindan los derechos más elementales a sus ciudadanos. Ante esto, lejos está el Episcopado a incitar a la rebelión violenta, más bien señalan que se trata de un impulso contrario a una vida cristiana. Sin embargo, comprenden que:

No debe, pues, extrañarnos que nazca en América Latina "la tentación de la violencia". No hay que abusar de la paciencia de un pueblo que soporta durante años una condición que difícilmente aceptarían quienes tienen una mayor conciencia de los derechos humanos. (SCGEL, 1968, p. 10) 
El mensaje es claro, no se justifica la violencia pero tampoco puede sorprender la reacción y organización del pueblo para terminar con las injusticias a las que es sometido. La postura del Vaticano es, como se presume, muy diferente a esta. Nos remitimos solamente a un pasaje de la Instrucción para ilustrarlo:

con frecuencia la aspiración a la justicia se encuentra acaparada por ideologías que ocultan o pervierten el sentido de la misma, proponiendo a la lucha de los pueblos para su liberación fines opuestos a la verdadera finalidad de la vida humana, y predicando caminos de acción que implican el recurso sistemático a la violencia, contrarios a una ética respetuosa de las personas. (Ratzinger, 1968, p.264)

Lo que menciona Ratzinger es destacable porque señala que el repudio a la violencia está en realidad orientado a ciertos grupos ideologizados que procuran la liberación de los pueblos de Latinoamérica. Si bien, la Iglesia ha tenido que manifestarse en contra de ciertas dirigencias estatales en América Latina, por su sistemática violación de los DD.HH.; coincidimos aquí con Berryman en que la mayoría de las veces, las posturas de la Iglesia o de los representantes con más jerarquía de la misma denuncian un lado de la violencia, mientras permanece en silencio cuando de denunciar a altos dirigentes se trata. Para Berryman, el caso de Nicaragua es paradigmático en este sentido. ${ }^{8}$ Desde esta perspectiva la Iglesia opta siempre por un tipo de actuación, denunciando la violencia de unos o eligiendo callar sobre la violencia de otros.

\section{Algunas reflexiones finales}

Hemos recorrido parte importante del pensamiento de los teólogos latinoamericanos y la teología de la liberación como un movimiento cristiano con un gran desarrollo y compromiso social y político. Mediante el análisis del Documento Conclusivo de Medellín analizamos algunas de las posturas presentes en ella, así como también las influencias que la hicieron posible y condicionaron su desarrollo.

\footnotetext{
${ }^{8}$ Al respecto señala " Así, los sacerdotes que colaboran con el gobierno sandinista son suspendidos, mientras el cardenal Obando, que denuncia a los sandinistas en cualquier oportunidad, pero nunca denuncia las atrocidades de los "contras" apoyados por Estados Unidos, y hasta celebra misas por sus partidarios en Miami, es envuelto con el manto del profeta." (Berryman, 1989, p.91)
} 
Por otro lado, mediante aportes de diversos autores y de un análisis contextualizado pudimos observar el desarrollo de la Teología de la Liberación y las tensiones que a nivel político se produjeron con el Vaticano, quien se mostró hostil a aceptar el fuerte compromiso político de la pastoral de la Teología de la Liberación.

Hemos señalado, además, los puntos que creemos resultan más relevantes de la Teología de la Liberación, que son a su vez los aspectos que más tensiones generan con la ortodoxia eclesial. Entre ellos observamos la opción preferencial por los pobres y oprimidos, la utilización de marcos de análisis de las cs. sociales, las tensiones en la visión dualista-trascendente de la historia, el marxismo, la cuestión del pecado, entre otros.

Finalmente intentamos brindar un aporte a la discusión mostrando lo que consideramos pueden ser los dos aspectos insalvables o más difíciles de conciliar entre la postura asumida por Ratzinger en nombre de la Sagrada Congregación para la doctrina de la fe y la postura que los teólogos de la liberación asumen en nombre de los pobres de Latinoamérica.

En este último punto, nos parece superadora la aceptación de que todo discurso y toda praxis está atravesada por elementos ideológicos. La cuestión estará en asumir o no esa influencia de manera crítica. En este sentido, nos parece que la postura de una supuesta neutralidad asumida por una de las partes, pierda solidez cuando se analizan los hechos de violencia que atraviesan y atravesaron los países latinoamericanos y las posturas que la jerarquía eclesial tomó al respecto.

En cuanto a la Teología de la Liberación, esta no ha podido siempre explicar bien como es concebida la cuestión de la liberación de los pueblos y su conciliación con la idea de una Trascendencia del Mundo de los Cielos, la conciliación entre la preferencia por los pobres y una eucaristía universal, entre otras cosas. Tal vez sus mayores aportes fueron asumir críticamente la tarea de la Iglesia como institución en América Latina, la revisión crítica de su historia y la influencia que tuvo en otros movimientos teológicos del mundo. 


\section{Referencias Bibliográficas}

- Berryman, P. (1989). Teología de la Liberación: Los hechos esenciales en torno al movimiento revolucionario en América Latina y otros lugares. México: Siglo veintiuno Editores.

- Consejo Episcopal Latinoamericano. (1968). Il Conferencia General del Episcopado Latinoamericano. Documentos Finales de Medellín. Recuperado de: http://www.celam.org/doc conferencias/Documento Conclusivo Medellin.pdf

- Cardoso, F.H y Falleto. E. (2011). Dependencia y desarrollo en América Latina: Ensayo de interpretación sociológica. Buenos Aires: Siglo veintiuno Editores.

- Gutierrez, G. (1975). Teología de la Liberación: Perspectivas. Salamanca: Ediciones Sígueme

- Ratzinger, J. (1984). Instrucción sobre algunos aspectos de la Teología de la Liberación. Encontrado en: https://www.cepchile.cl/cep/estudios-publicos/n-1a-la-30/estudios-publicos-n-17/instruccion -sobre-algunos-aspectos-de-lateologia-de-la-liberacion

- Scannone, J.C. (1982). La teología de la liberación. Caracterización, corrientes, etapas. Stromata, $38 . \quad$ Encontrado https://seleccionesdeteologia.net/selecciones/llib/vol23/92/092_scannone.pdf 